\title{
Multi-site study of HPV type-specific prevalence in women with cervical cancer, intraepithelial neoplasia and normal cytology, in England
}

\author{
R Howell-Jones', A Bailey², S Beddows ${ }^{3}$, A Sargent ${ }^{2}$, N de Silva ${ }^{3}$, G Wilson ${ }^{4}$, J Anton ${ }^{3}$, T Nichols ${ }^{5}$, K Soldan ${ }^{*, 1}$ \\ and $\mathbf{H}$ Kitchener $^{6}$ on behalf of the Study Group Collaborators ${ }^{7}$
}

'HIV \& STI Department, Health Protection Agency Centre for Infections, 61 Colindale Avenue, London NW9 5EQ, UK; ${ }^{2}$ Department of Clinical Virology, Central Manchester NHS Foundation Trust, Second Floor Clinical Sciences Building 2, Manchester Royal Infirmary, Oxford Road, Manchester M 3 9WL, UK; ${ }^{3}$ Virus Reference Department, Health Protection Agency Centre for Infections, 6 I Colindale Avenue, London NW9 5EQ, UK; ${ }^{4}$ Department of Histopathology, Central Manchester NHS Foundation Trust, Second Floor Clinical Sciences Building 2, Manchester Royal Infirmary, Oxford Road, Manchester M 3 9WL, UK; ${ }^{5}$ Statistics, Modelling and Bioinformatics Department, Health Protection Agency Centre for Infections, 61 Colindale Avenue, London NW9 5EQ, UK; ${ }^{6}$ School of Cancer and Enabling Sciences, University of Manchester, Manchester MI 3 9WL, UK

\begin{abstract}
BACKGROUND: Knowledge of the prevalence of type-specific human papillomavirus (HPV) infections is necessary to predict the expected, and to monitor the actual, impact of HPV immunisation and to design effective screening strategies for vaccinated populations. METHODS: Residual specimens of cervical cytology $(N=47 / 9), C I N 3 / C G I N$ and cervical cancer biopsies $(N=15$ I5) were obtained from sites throughout England, anonymised and tested for HPV DNA using the Linear Array typing system (Roche).

RESULTS: The prevalence of HPV 16 and/or 18 (with or without another high-risk (HR) type) was 76\% in squamous cell carcinomas, 82\% in adeno/adenosquamous carcinomas and $63 \%$ and $91 \%$ in CIN3 and CGIN, respectively. Of all HR HPV-infected women undergoing cytology, non-vaccine HPV types only were found in over $60 \%$ of those with mild dyskaryosis or below, and in $<20 \%$ of those with cancer. In women of all ages undergoing screening, HR HPV prevalence was $16 \%$ and HPV 16 and/or 18 prevalence was $5 \%$.

CONCLUSION: Pre-immunisation, high-grade cervical disease in England was predominantly associated with HPV 16 and/or 18 , which promises a high impact from HPV immunisation in due course. Second-generation vaccines and screening strategies need to consider the best ways to detect and prevent disease due to the remaining HR HPV types.

British Journal of Cancer (2010) 103, 209-216. doi: I0.1038/sj.bjc.6605747 www.bjcancer.com

(c) 2010 Cancer Research UK
\end{abstract}

Keywords: human papillomavirus (HPV); cervical cancer; cervical screening; immunisation

A wide range of international studies have found approximately $70 \%$ of cervical cancers to be associated with human papillomavirus (HPV) types 16 or 18 , and therefore preventable by currently available HPV vaccines (Clifford et al, 2003; Muñoz et al, 2004). The United Kingdom introduced an HPV immunisation programme in September 2008 and expects to see a consequent decline in cervical cancers and cervical intraepithelial neoplasia in the coming decades (Choi et al, 2009).

In 2006, almost 3000 women were diagnosed with cervical cancer in the United Kingdom, and there were around 1000 deaths (Cancer Research UK, 2009). It has been estimated that cervical screening prevents at least $70 \%$ of cases that would occur without screening (Sasieni et al, 2003). To achieve this, the NHS Cervical Screening Programme (NHS CSP) in England screens more than 3 million women aged 25-64 years annually and refers more than 100000 to colposcopy examination (The NHS Information Centre, 2009).

Several studies have investigated HPV prevalence in women attending for cervical screening in the United Kingdom (Cuschieri et al, 2004; Kitchener et al, 2006; Hibbitts et al, 2008) and have provided valuable

\footnotetext{
*Correspondence: Dr K Soldan; E-mail: Kate.Soldan@hpa.org.uk

${ }^{4}$ See Appendix for Study Group Collaborators.

Received 9 March 20 I0; revised 10 May 20 I0; accepted 26 May 2010
}

data regarding the frequency of HPV infection. These studies have been conducted in specific areas of the country, at different times, using different testing methods, and as they have sampled all women attending for screening they have inevitably provided fewer data regarding the rarer disease-grades, and some age-groups.

Better knowledge of HPV type-specific prevalence and distribution at each stage of cervical disease and among women of different ages would both improve estimates of the expected impact from HPV $16 / 18$ immunisation and inform planning of possible future screening services, particularly those using HPV testing.

The aim of this study was to provide robust baseline estimates of the prevalence and distribution of HPV types among a nationally representative sample of women, for the full age range undergoing screening (25-64 years) and for each disease phase (from normal cytology to cervical cancer), prior to any impact of the National HPV Immunisation Programme.

\section{MATERIALS AND METHODS}

\section{Sample collection and processing}

Two sample types were included in the study: (i) tissue sections from routinely obtained diagnostic biopsies of cervical cancers and 
high-grade precancerous lesions archived in NHS pathology laboratories, and (ii) residual liquid-based cytology (LBC) samples from women attending for cervical screening. Samples were obtained from eight participating NHS pathology laboratories in England. Three laboratories (Gateshead Health NHS Foundation Trust, Birmingham Women's NHS Foundation Trust and Royal Free Hampstead NHS Trust (London)) provided both tissue sections and LBC samples, three laboratories (Central Manchester University Hospitals NHS Foundation Trust, Sheffield Teaching Hospitals NHS Foundation Trust and Barts and The London NHS Trust) provided tissue sections only and two laboratories (Gloucestershire Hospitals NHS Foundation Trust and Norfolk and Norwich University Hospitals NHS Foundation Trust) provided LBC samples only.

Cancer and CIN3/CGIN biopsies Sections from archived blocks of cervical intraepithelial neoplasia 3 (CIN3), cervical glandular intraepithelial neoplasia (CGIN), squamous cell carcinoma (SCC) and adenocarcinoma (including adeno-squamous carcinoma) (ADC) tissue were collected from the six participating sites between June 2006 and July 2008. Cases were deemed suitable for inclusion in the study after confirmation of the pathological diagnosis by an experienced subspecialist gynaecological histopathologist at each of the six participating centres. The biopsies of cancer had been originally embedded between 1986 and 2008; 96\% had been embedded since 2000. The CIN3 and CGIN biopsies had been collected between 2000 and 2008. Approximately six $10 \mu$ thick sections from each tissue block were placed in a $1.6 \mathrm{ml}$ Eppendorf tube. A further section was stained and re-examined by the pathologist to confirm the presence of diseased tissue. To prevent cross-contamination from residual wax from the previous block, the microtome blade was cleaned with ethanol between blocks. Tissue sections were sent to the Department of Clinical Virology, Central Manchester University Hospitals NHS Foundation Trust for HPV genotyping. The following data were collected on a study record for each sample and sent to Manchester: month and year of birth, date of sample collection, type of lesion (CIN3, CGIN or cancer) and histology result.

Tissue sections were de-waxed by adding a $1 \mathrm{ml}$ of octane to each Eppendorf tube followed by $75 \mu \mathrm{l}$ of methanol. Tubes were vortexed and incubated at $56^{\circ} \mathrm{C}$ for $30 \mathrm{~min}$ followed by centrifugation at 13000 r.p.m. for $1 \mathrm{~min}$ after which the octane layer was removed using a fine-tipped Pasteur pipette. The tissue pellet was then washed with $1.0 \mathrm{ml}$ of ethanol, centrifuged as before and the ethanol removed. The tubes were then left at $56^{\circ} \mathrm{C}$ for 30-45 min to evaporate off residual ethanol. The tissue was digested in $600-1000 \mu \mathrm{l}$ (depending on size of pellet) of proteinase $\mathrm{K}$ lysis buffer containing $10 \mathrm{~mm}$ Tris/ $\mathrm{HCl}$ buffer $\mathrm{pH} 8.3 ; 1 \mathrm{~mm}$ EDTA, $0.5 \%$ Triton X-100, $0.002 \%$ SDS and $250 \mu \mathrm{g} \mathrm{ml}^{-1}$ of proteinase K. Tissue digestion was carried out at $56^{\circ} \mathrm{C}$ for $72 \mathrm{~h}$ with constant agitation. DNA was then extracted from a $200 \mu \mathrm{l}$ aliquot of digested tissue using the Roche MagNA Pure (Roche Applied Science, Penzberg, Germany) automated extraction system with an elution volume of $100 \mu \mathrm{l}$.

LBC samples Residual LBC samples were collected prospectively from women undergoing cervical screening between October 2007 and January 2009 at five participating sites. Stratified sampling was used to obtain sufficient high-risk (HR) HPV-positive samples to analyse type distribution within each combination of age-band and cytology grade, thus a disproportionately higher number of older women and those with more severe cytology outcomes were sampled. Before inclusion in the study, samples were collected and handled according to local protocols: Thinprep and Surepath LBC systems were both in use, with samples stored at ambient temperatures while awaiting cytological examination. After completion of cytology, residual LBC samples that matched an unmet age-group and grade (according to the site's stratified sampling frame) were anonymised and sent to the Health Protection Agency (HPA) Virus Reference Department for HPV genotyping. For Surepath samples, the tubes (enriched sample), not the vials, were used as the vials were found to have an inadequate amount of cellular material. The tube approximated to levels found using Thinprep, although with more variability. The following data were collected on a study record for each sample and sent to the HPA: month and year of birth, cytology result, date of sample, outward postcode (i.e., up to the first four characters), biopsy taken (and histology result if applicable).

$1 \mathrm{ml}$ of each LBC sample was centrifuged for $5 \mathrm{~min}$ at 13000 r.p.m. and the cellular pellet suspended in $300 \mu \mathrm{l}$ of cold sterile phosphate-buffered saline. After a lysis step (addition of $40 \mu \mathrm{l}$ Qiagen Protease and $265 \mu \mathrm{l}$ Qiagen Buffer AL containing guanidine hydrochloride) samples were stored at $-25^{\circ} \mathrm{C}$ before nucleic acid extraction. Nucleic acid extraction was conducted on the BioRobot Universal platform (Qiagen, Crawley, West Sussex, UK) using the QIAamp DNA Blood BioRobot MDx kit and the extraction protocol QIAamp 'One for All UNIV rcV23'.

\section{HPV Genotyping}

A $50 \mu \mathrm{l}$ aliquot of the extracted DNA from the biopsy samples and a $10 \mu \mathrm{l}$ aliquot of the extracted DNA from the LBC samples (i.e., approximately $10 \%$ of the original $1 \mathrm{ml}$ material, equal to the suggested input for the Roche Linear Array (LA) test (Roche Molecular Systems, Inc., Branchburg, NJ, USA)) was used for the PCR reaction stage of the Roche LA test. The Roche LA test was used according to manufacturer's instructions, with the exception of the sample extraction methods described above. Individual oligonucleotide capture probes enabled identification of $37 \mathrm{HR}$ and low-risk HPV genotypes and the $\beta$-globin amplicon acted as a control for cell adequacy, extraction and amplification. Identification of HPV 52 in this system was possible by the use of a cross-reactive oligonucleotide probe $(52 \mathrm{M})$ that hybridises with HPV genotypes $33,35,52$ and 58. The presence of HPV 52 was therefore inferred by reactivity to the $52 \mathrm{M}$ probe with no reactivity to the individual oligonucleotide probes representing HPV 33, 35 and 58. The presence of a co-infection of 52 with 33,35 or 58 would not have identified HPV 52. The HPV-positive and -negative controls provided within the Roche LA test kit were included for every 46 tissue section samples and every 22 LBC samples tested. The time from LBC sample collection to receipt at HPA (median 27 days, inter-quartile range $21-35)$ was not negatively associated with detection of $\beta$-globin or HPV DNA: this variable was not considered further.

Following an assessment of type-specific assay reproducibility (details in Supplementary online material) only those bands that gave a signal equal to or greater than the $\beta$-globin low control band were recorded.

For tissue section samples only, additional analysis using the Inno-Lipa Extra (Microgen Bioproducts Ltd, Camberley, Surrey, UK) genotyping assay was performed on those samples that were $\beta$-globin negative or in which no HR HPV DNA was detected using Roche LA test (Figure 1). The Inno-Lipa assay is a similar format line blot assay to Roche LA (and also includes a $\beta$-globin control), however, it amplifies a much smaller fragment of the DNA than the Roche LA assay making it less likely to be affected by DNA fragmentation, which may occur during tissue fixation. A $5 \mu \mathrm{l}$ aliquot of extracted DNA was amplified and detected according to manufacturer's instructions.

Figure 1 shows the flow of tissue sections and LBC samples included in the study.

\section{Data analysis}

A valid result was defined as any sample with a positive $\beta$-globin result by either Roche LA or Inno-Lipa Extra. Only samples with a valid HPV typing result $(N=6234$, Figure 1$)$ were included in the 


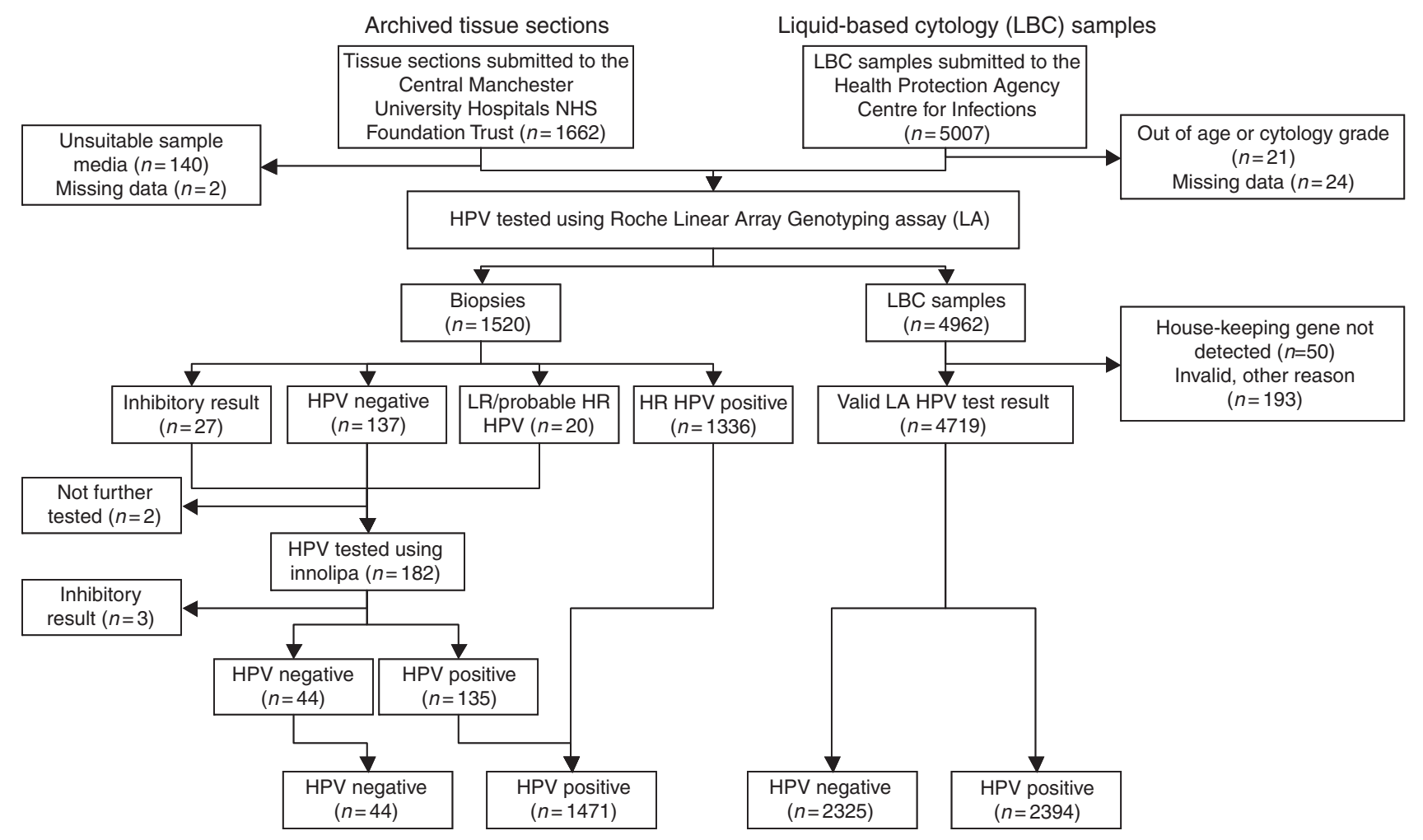

Figure I Sample submission and human papillomavirus (HPV) testing algorithm for archived tissue sections and residual liquid based cytology (LBC) samples. Abbreviations: $H R=$ high-risk; $L R=$ low-risk.

analysis. HR HPV types were defined according to the 2009 International Agency Research on Cancer classification of types, which were at least 'probably carcinogenic to humans', that is, HPV 16, 18, 31, 33, 35, 39, 45, 51, 52, 56, 58, 59 and 68 (Bouvard et al, 2009). Five groups of HPV types were defined for the purpose of the analysis: (i) HPV 16 and/or HPV 18 irrespective of other types, (ii) HPV 16 and/or 18 without any other HR types, (iii) HPV 16 and/or 18 with other HR type(s) (iv) HR HPV (any one or more type(s)) but not HPV 16 or HPV 18 and (v) any HR HPV infection. The prevalence of HPV infection, for each group above, for each age-group and disease-grade, was calculated with $95 \%$ confidence intervals (CIs) that allowed for the samples being effectively clustered through the collection from a sample of laboratories. For the LBC samples, prevalence estimates, other than those for a particular age-band and cytology grade, were calculated using sampling weights based on the number of women attending for cervical screening as part of the NHS CSP in 2007-2008. This was necessary to estimate prevalence for the screened population as our stratified sampling purposely selected disproportionate numbers from each age-band and cytology-grade. To analyse prevalence in each region, sampling weights (based on national cervical screening data as described above) were used and applied to samples from each laboratory separately. Differences in prevalence by region were assessed using Pearson's $\chi^{2}$ statistic.

\section{RESULTS}

Valid data and HPV results were obtained for 1515 biopsy sections and 4719 LBC samples submitted to the study. The biopsy sections consisted of 906 CIN3, 450 SCC, 54 CGIN and 105 ADC samples (including 89 adeno- and 16 adenosquamous-carcinomas), while the LBC samples were from 2452 women without cervical disease, 1051 with borderline, 697 with mild, 276 with moderate and 243 with severe dyskaryosis. The mean (s.d.) age of the women at biopsy was 31.9 (8.1), 49.7 (16.1), 35.4 (7.8) and 45.5 (14.1) years for CIN3, SCC, CGIN and ADC, respectively. The numbers of samples included in the study, by participating site, are available in Supplementary Table S1.

\section{HR and HPV 16 and/or 18 prevalence}

The prevalence of each of our defined categories of HR HPV, by age-group and disease-grade, from normal to cancer, are shown in Table 1. Figure 2 shows HPV 16 and/or 18 infections by diseasegrade in each age-band, while additional data by disease-grade in each age-band are available in Supplementary Table S2. HR HPV types were detected in $95.8 \%$ (95\% CI $94.4-96.8 \%)$ and $95.2 \%$ (95\% CI 84.8-98.6\%) of SCC and ADC, respectively. HPV 16 and/or 18 (alone or with other HR types) were detected in $76.4 \%$ $(95 \%$ CI $71.0-81.1 \%)$ and $81.9 \%$ (95\% CI $73.2-88.2 \%)$ of SCC and ADC, respectively, while HPV 16 and/or 18 were the only HR types detected in $72.0 \%$ (95\% CI $67.3-76.3 \%)$ and $75.2 \%$ (95\% CI $64.3-83.7 \%$ ) of SCC and ADC, respectively (Table 1).

In biopsies from high-grade cervical abnormalities, HR HPV were detected in $94.2 \%$ (95\% CI $87.7-97.3 \%)$ and $98.1 \%$ (95\% CI $86.4-99.8 \%$ ) of CIN3 and CGIN, respectively. HPV 16 and/or 18 were detected in $63.2 \%$ (95\% CI $57.0-69.1 \%)$ and $90.7 \%$ (95\% CI $85.0-94.4 \%$ ) of CIN3 and CGIN, respectively, and were the only HR types detected in $53.9 \%$ (95\% CI $48.7-59.0 \%$ ) and $83.3 \%$ (95\% CI 61.1-94.1\%), of CIN3 and CGIN, respectively (Table 1).

In women of all ages undergoing screening, the prevalence of HR HPV and of HPV 16 and/or 18 was $15.7 \%$ (95\% CI $11.2-21.6 \%$ ) and $5.1 \% \quad(95 \%$ CI $3.3-8.0 \%)$, respectively (Table 1$)$. The prevalence of HR HPV and HPV 16 and/or 18 increased with increasing severity of cytology grade, and was higher among younger women, within all cytology grades (more markedly in the lower grades) (Figure 2). Among residual LBC samples from women with severe dyskaryosis the prevalence of HR HPV ranged from $86 \%$ in 50 - to 64 -year olds to $97 \%$ in 25 - to 29 -year olds. 
Table I Prevalence of high risk (HR) HPV types by age-band and cervical grade for the screened population (overall, by age and by disease grade) and for women with severe cervical abnormalities and cervical cancer.

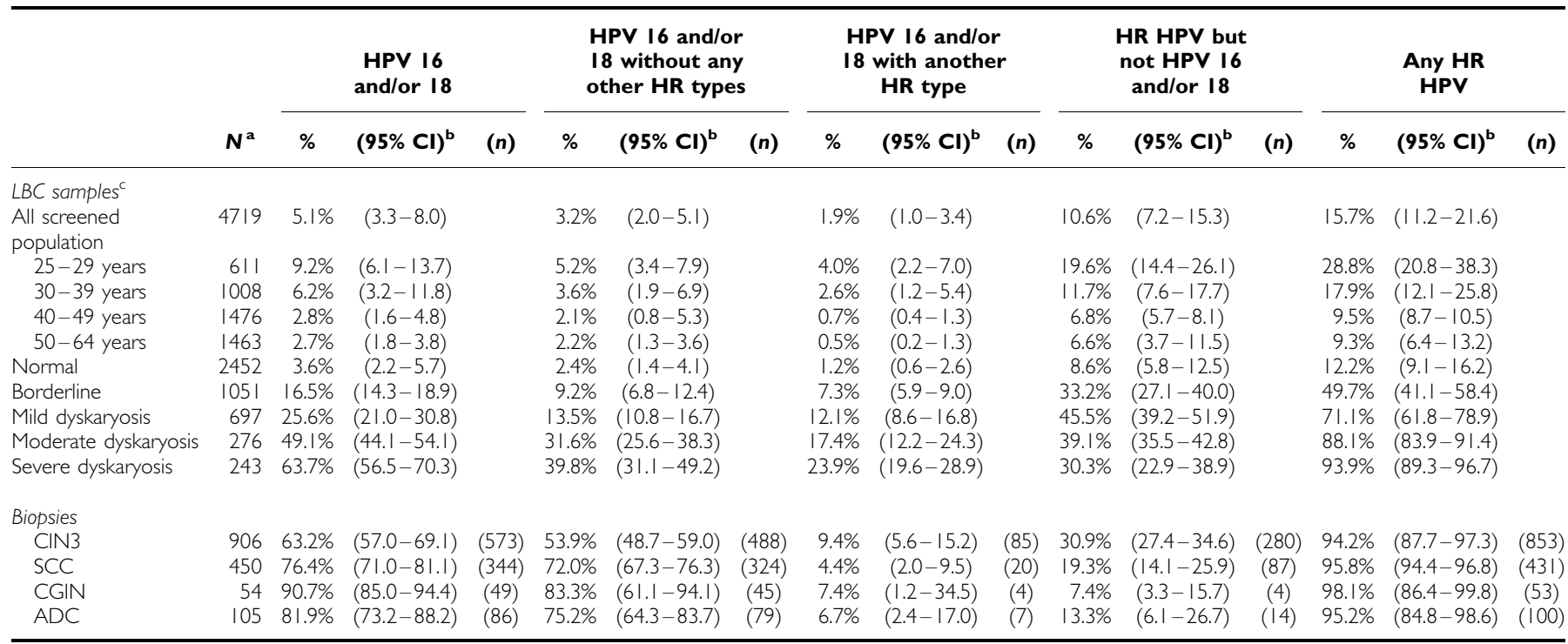

Abbreviations: $\mathrm{ADC}=$ adeno and adeno-squamous carcinoma; $\mathrm{Cl}=$ confidence interval; $\mathrm{CIN3}=$ cervical intraepithelial neoplasia 3; $C \mathrm{CIN}=$ cervical glandular intraepithelial neoplasia; HPV = human papillomavirus; HR = high-risk; LBC = liquid-based cytology; SCC = squamous cell carcinoma. ${ }^{\mathrm{a}}$ |6I LBC samples, I33 CIN3, 2 CGIN, 5 SCC and 2 ADC from women aged $<25$ years. ${ }^{\circ} 95 \%$ Cls were calculated allowing for samples being clustered within laboratories. Weighted to allow for disproportionate LBC sample collection by age and/or cytology grade as appropriate.

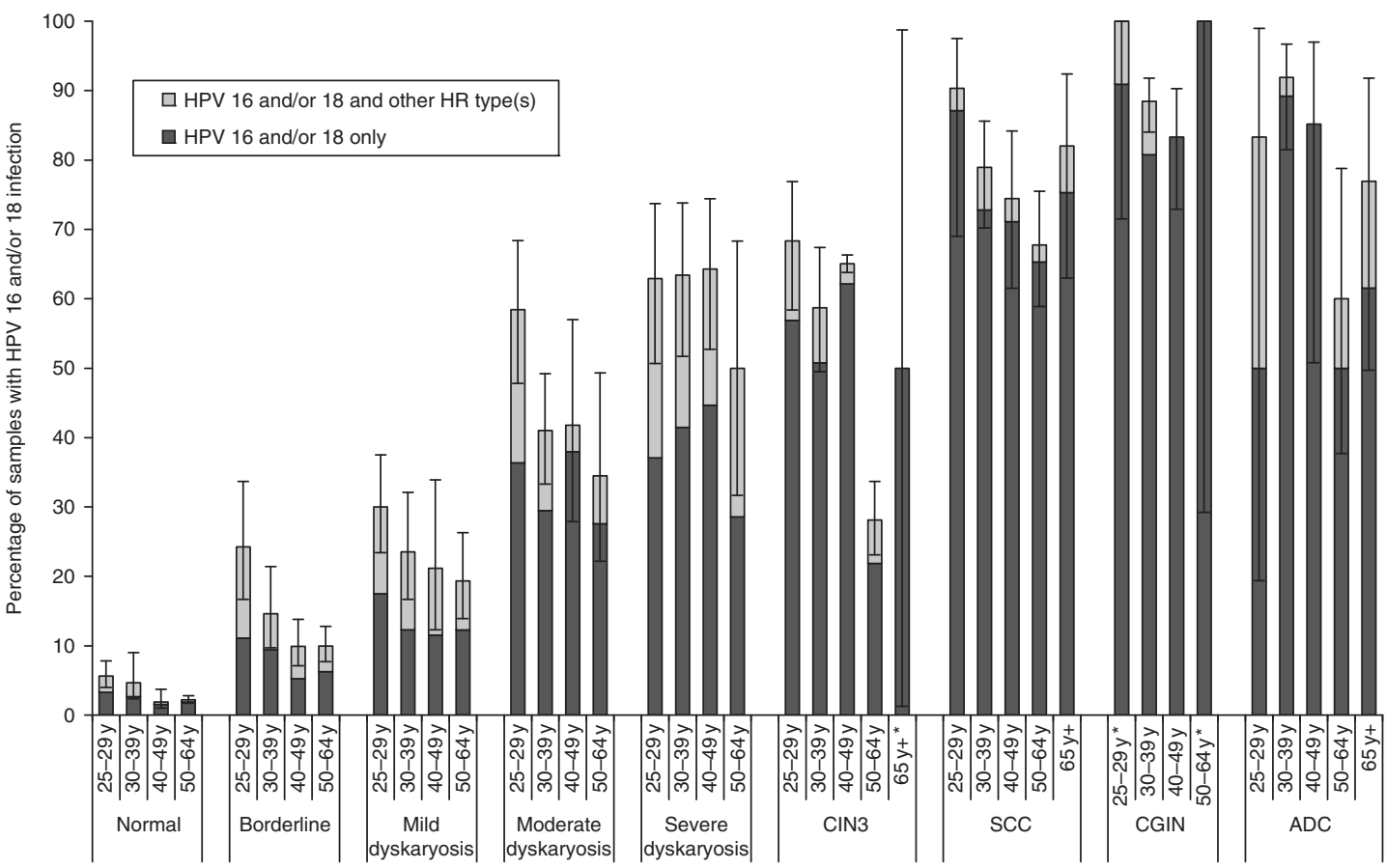

Figure 2 Prevalence of human papillomavirus (HPV) 16 and/or 18 (alone and in mixed infections with other high-risk (HR) HPV types) by cervical grade and age band. 95\% confidence intervals were calculated allowing for samples being clustered within laboratories, except where these could not be determined because of there being no variation between laboratories (i.e. all I00\% prevalence) or when all samples were from one laboratory (i.e. CIN3 samples from women aged $65+$ years), when confidence intervals (one-sided $97.5 \%$ where appropriate) were calculated without allowing for clustering (*). Abbreviations: $A D C=$ adeno- and adeno-squamous carcinoma; CIN3=cervical intraepithelial neoplasia 3; CGIN=cervical glandular intraepithelial neoplasia; SCC = squamous cell carcinoma.

The prevalence of HPV 16 and/or 18 in severe dyskaryosis alone or with other HR types showed no strong trend with age, at between 50 and 64\%, with HPV 16 and/or 18 alone (without other HR types) detected in between 29 and $45 \%$ (Figure 2). The crude prevalence of HR HPV and of HPV 16 and/or 18 infection in the LBC samples from women confirmed on histology examination to have CIN3 disease was $95.0 \%(n=226)$ and $62.6 \%(n=149)$, respectively. Women of all ages with moderate dyskaryosis also 

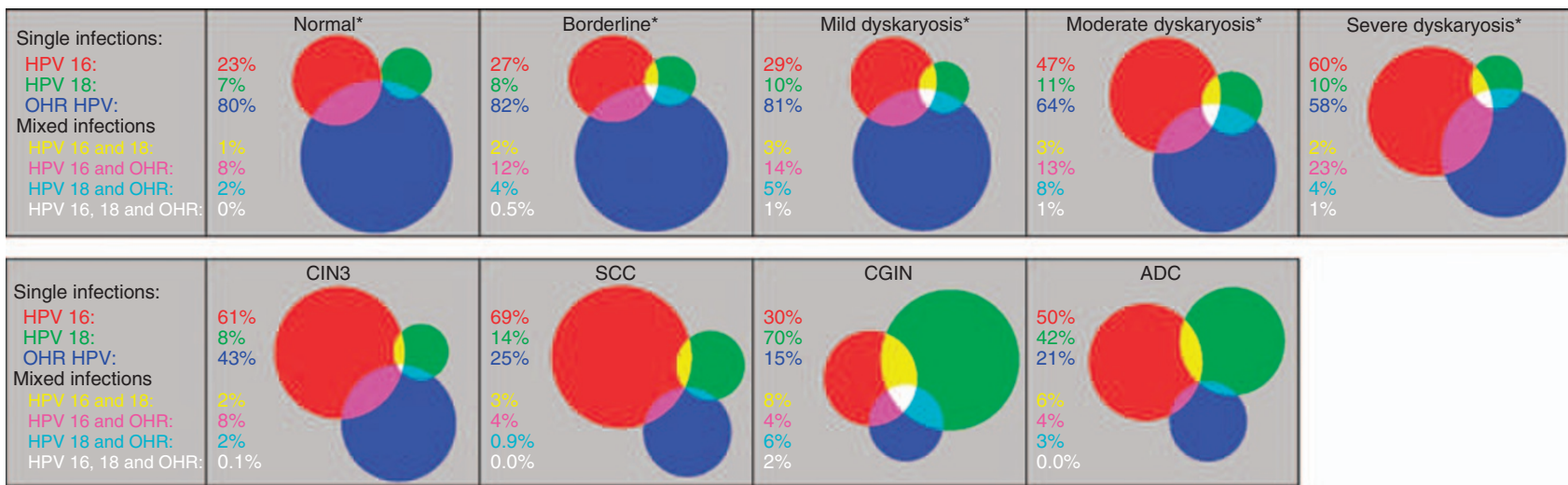

Figure 3 Proportional Venn diagrams showing human papillomavirus (HPV) 16, HPV I8 and high-risk (HR) types other than HPV I6 or HPV I8 (OHR) in HR HPV-positive samples, by cervical grade (Chow and Rodgers, 2005). Red: HPV I6; green: HPV I 8; blue: OHR; yellow: HPV I6 and HPV I 8; pink: HPV 16 and OHR; turquoise: HPV 18 and OHR; white: HPV 16, 18 and OHR. *Age-weighted percentages (to allow for disproportionate liquid-based cytology sample collection by age). Abbreviations: $A D C=$ adeno- and adeno-squamous carcinoma; CIN3 = cervical intraepithelial neoplasia 3; CGIN= cervical glandular intraepithelial neoplasia; SCC = squamous cell carcinoma.

Table 2 HR and HPV 16 and/or 18 prevalence by region (submitting laboratory) in women undergoing cervical screening

\begin{tabular}{|c|c|c|c|c|}
\hline \multirow[b]{2}{*}{ Submitting laboratory } & \multirow[b]{2}{*}{ Sample type } & \multirow[b]{2}{*}{$N(\%)$} & \multicolumn{2}{|c|}{ Prevalence $^{\mathrm{a}}(95 \% \mathrm{Cl})$} \\
\hline & & & HR HPV & HPV 16 and/or 18 \\
\hline Gateshead (GH) & Surepath & $1063(22.5)$ & $16.9(\mid 4.3-19.9)$ & $4.2(3.0-5.9)$ \\
\hline Gloucestershire (GL) & Thinprep & $927(19.6)$ & $13.3(10.4-16.9)$ & $6.4(4.6-6.6)$ \\
\hline Norfolk (NN) & Thinprep & $1077(22.8)$ & $16.2(13.4-19.4)$ & $6.2(4.6-8.4)$ \\
\hline London (RF) & Thinprep & $633(13.4)$ & $\begin{array}{c}20.1(15.1-26.2) \\
P=0\end{array}$ & $6.5(3.8-10.8)$ \\
\hline
\end{tabular}

Abbreviations: BW = Birmingham Women's NHS Foundation Trust; Cl= confidence interval; GH = Gateshead Health NHS Foundation Trust; GL = Gloucestershire Hospitals NHS Foundation Trust; NN = Norfolk and Norwich University Hospitals NHS Foundation Trust; RF = Royal Free Hampstead NHS Trust (London). ${ }^{a}$ All prevalence estimates weighted for each laboratory to allow for differences between age and cytology distribution in study sample vs national population. ${ }^{b}$ Pearson's $\chi^{2}$.

had high prevalence of HR HPV and of HPV 16 and/or 18 infection (69-94\% and $35-58 \%$, respectively) (Figure 2 and Supplementary Table S2). With increasing disease-grade, there was an increasing proportion of HPV 16 and HPV 18 in HPV-positive samples, and a concomitant decrease in other HR HPV types (Figure 3).

Prevalence of HR HPV by region ranged from $13.3 \%$ in Gloucestershire to $20.1 \%$ in London, while prevalence of HPV 16 and/or 18 ranged from $4.2 \%$ in Gateshead to $6.5 \%$ in London (Table 2). Differences in prevalence by region were not statistically significant (Pearson's $\left.\chi^{2} P>0.05\right)$. Further exploration of HR HPV prevalence in young women (25-29 years) with normal cytology results also found no significant differences between regions (data not shown).

\section{Type-specific prevalence}

HPV 16 was the most commonly detected type in all cervical grades apart from CGIN wherein HPV 18 was the most commonly detected type. The four next most frequently identified HPV types in the SCC biopsy samples (in single or multiple infections) were, in descending order, HPV 33, 45, 52 and 31. The prevalence of these six types in cancer, precancerous lesions and normal cytology samples is shown in Figure 4. The six most common HPV infections in women with normal cytology were, in descending order, HPV 16, 61, 62, 53, CP6108 and 54; HPV 18 was the nineteenth most common type (HPV type-specific prevalence by grade of disease is available in Supplementary Table S3).

The four HR types found most commonly in SCC biopsy samples, after HPV 16 and 18, showed a general pattern of increasing prevalence with increasing grade of disease although all, except HPV
45, had higher prevalence in severe grade lesions and CIN3 than in cervical cancers (Figure 4). The age-weighted prevalence of HPV 31 and 52 peaked in samples with moderate dyskaryosis (Figure 4).

The prevalence, in the absence of HPV 16 or 18, in SCC and ADC of selected HPV types from the A9 and A7 species, closely related to HPV 16 and 18 respectively, is shown in Table 3 . The five types with emerging evidence of cross-protection from HPV 16/18 vaccines (HPV 31, 33, 45, 52 or 58 ) were found in $14.7 \%$ of SCC and $10.5 \%$ of ADC in the absence of HPV 16 and 18 .

HPV 6 and/or 11 were detected in very few LBC samples without HR types $(n=46,0.8 \%$ weighted prevalence). Of these samples, $34.8 \%(n=16)$ had normal cytology, 30.4\% $(n=14)$ had borderline and $34.8 \%(n=16)$ had mild dyskaryosis. No women with moderate or severe dyskaryosis were found to have HPV 6 and/or 11 without a HR HPV type. Overall (irrespective of other types), the age-weighted (to NHS CSP population) prevalence of HPV 6 and/or 11 was $0.9 \%(95 \%$ CI $0.6-1.5 \%)$ in normal samples, $0.1 \%$ (95\% CI $0.08-0.14 \%)$ in borderline samples, $0.1 \%$ (95\% CI $0.05-$ $0.3 \%)$ in mild dyskaryotic samples, $0.02 \%(95 \%$ CI $0.01,0.1 \%)$ in moderate dyskaryotic and $0.01 \%(95 \%$ CI $0.0,0.04 \%)$ in severe dyskaryotic samples. In women with normal cytology, the weighted prevalence of HPV 6 was $0.8 \%$ and HPV 11 was $0.2 \%$ (Supplementary Table S3).

\section{DISCUSSION}

We have used anonymous, residual, cervical cytology (LBC) samples and biopsies of cervical cancers and high-grade lesions, collected from eight sites, to describe type-specific HPV prevalence 


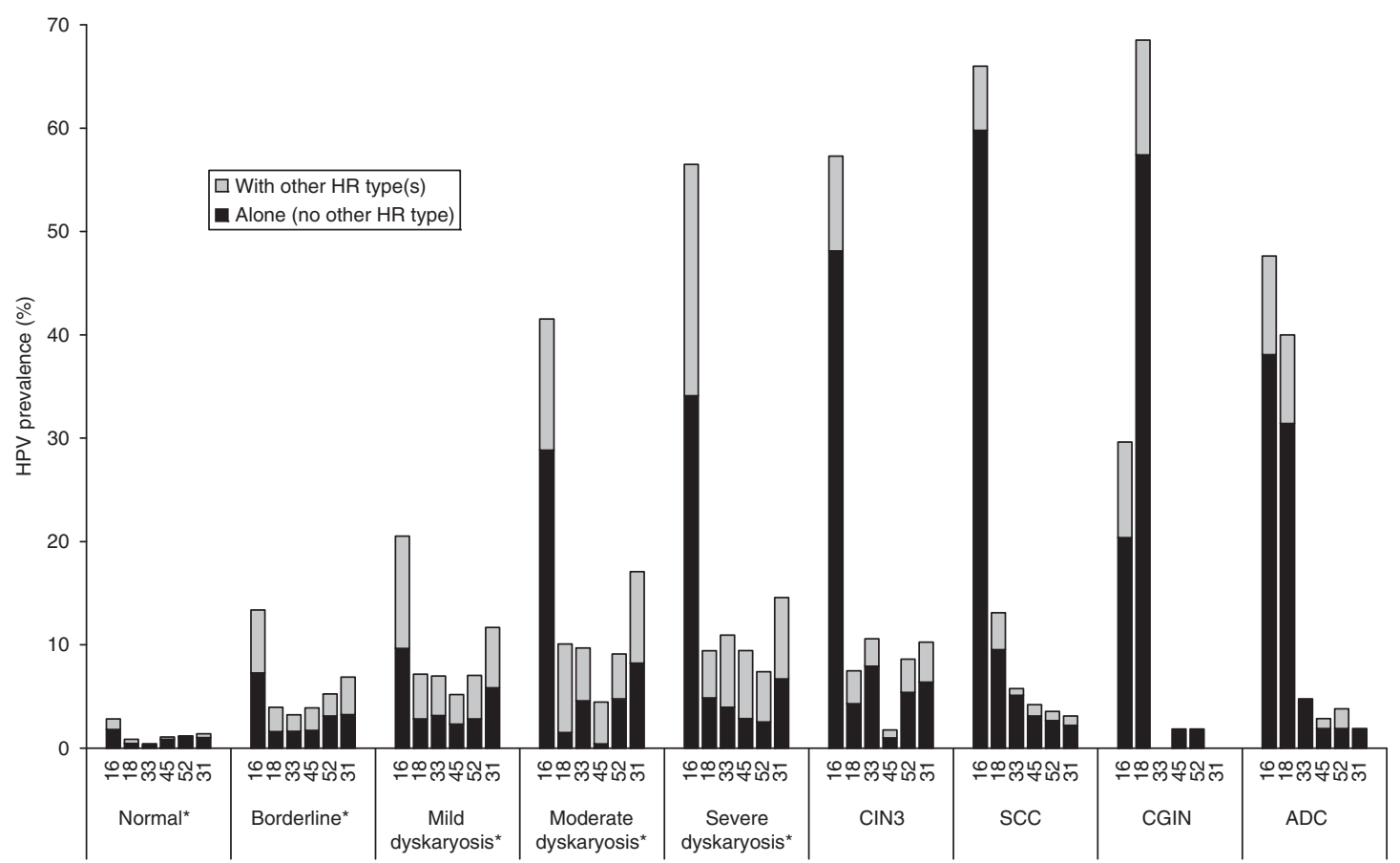

Figure 4 Human papillomavirus (HPV) prevalence by cervical grade of the six most common types found in squamous cell carcinoma (SCC) biopsies. *Age-weighted prevalence (to allow for disproportionate liquid-based cytology sample collection by age). Abbreviations: ADC =adeno- and adenosquamous carcinoma; CIN3 = cervical intraepithelial neoplasia 3; CGIN=cervical glandular intraepithelial neoplasia.

Table 3 Prevalence of selected A9 and A7 types in squamous cell carcinoma (SCC) and adeno- and adeno-squamous carcinoma (ADC), with and without human papillomavirus (HPV) 16 and 18

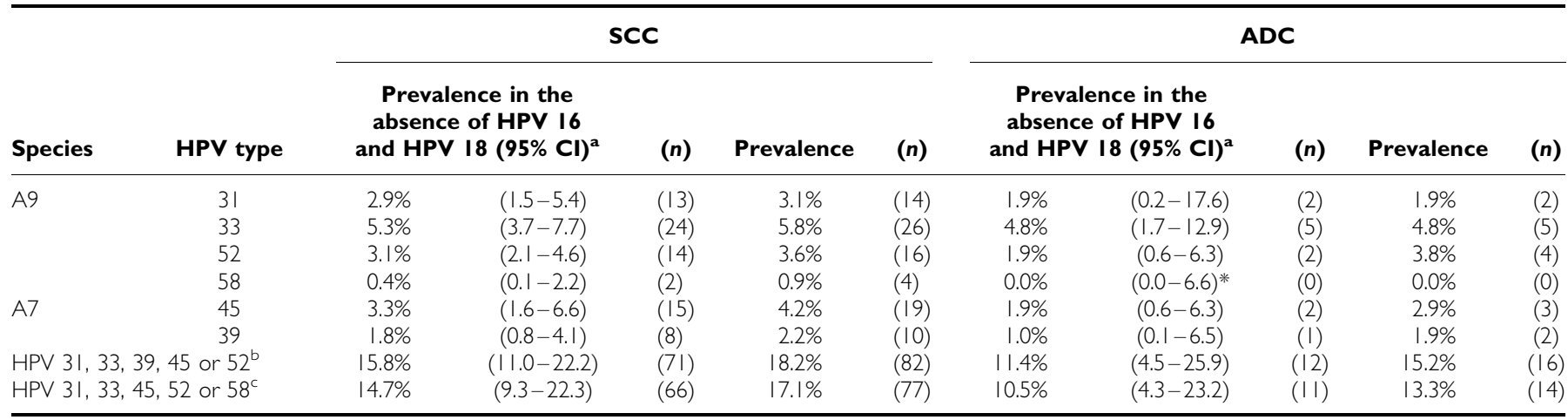

a95\% confidence intervals were calculated allowing for samples being clustered within laboratories except where these could not be determined because of no variation between laboratories (i.e., all 0\% prevalence), when one-sided $97.5 \%$ confidence intervals have been calculated (*). ' ${ }^{\text {bive }}$ most common types in SCC after HPV I6 and I8. ${ }^{\mathrm{C}}$ Types against which some cross-protection efficacy results have been reported from clinical trials (Paavonen et al, 2009; Brown et al, 2009).

in women in England, across the full spectrum of cervical pathology, from normal to cancer.

Assuming the relative frequency of SCC and ADC in our study are representative of all cervical cancers, our results suggest that at least $73 \%$ of cervical cancers in England are potentially preventable by type-specific protection from HPV 16/18 immunisation (i.e., are infected with HPV 16 and/or 18 only), rising to around $77 \%$ if co-infection with other HR types is never the causative HPV infection. Similarly, at least $56 \%$ and up to $65 \%$ of CIN3/CGIN are potentially preventable by HPV $16 / 18$ immunisation. The difference between the percentage of cases with HPV 16 and/or 18 only (minimum attributable fraction), and the percentage with HPV 16 and/or 18 and another HR HPV (probable attributable fraction), indicates the potential for unmasking of disease caused by other HR HPV types if HPV 16 and 18 infections are prevented. By the same reckoning, between 32 and $49 \%$ of the moderate and 40 and $64 \%$ of the severe dyskaryotic cervical abnormalities identified by cervical screening may be preventable by HPV 16/18 immunisation.

HPV 31 and 45 were each associated (without HPV 16 and/or 18, but with or without other HR types) with an additional 3\% of SCC and $2 \%$ of ADC. As there is some evidence of cross-protection from current HPV 16/18 vaccines against infection and disease due to these types and HPV 33, 52 and 58, which are closely related to HPV 16 or HPV 18 (Brown et al, 2009; Paavonen et al, 2009), the benefits of immunisation may include reduction in these cancers also. If cross-protection against HPV 31, 33, 45, 52 and 58 ranges from 25 to $29 \%$, as suggested currently by clinical trials (Brown et al, 2009; Paavonen et al, 2009), our findings suggest that HPV $16 / 18$ immunisation may prevent an additional $3-4 \%$ of cervical cancers in England.

Other studies of HPV type distribution in cervical cancers in the United Kingdom have reported similarly high fractions of cases probably attributable to HPV 16 and/or 18. Cuzick et al (2000) 
analysed cervical scrapes from 116 women with cervical cancer and found HPV 16 and/or 18 in 78\% of SCC and 71\% of ADC (including adenocarcinoma and adeno-squamous carcinoma). In Scotland, Tawfik El-Mansi et al (2006) found HPV 16 and/or 18 in $61 \%$ of ADC diagnosed between 1991 and 2001, and Cuschieri et al (2010) detected HPV 16 and/or 18 in $72 \%$ of 370 invasive cervical cancers diagnosed up to 2004. In Wales, Powell et al (2009) found HPV 16 and/or 18 in $80 \%$ of SCC $(N=222)$ and $91 \%$ of ADC $(N=47)$ diagnosed between 2000 and 2006. Three other studies have looked at fewer than 50 cases each (Crook et al, 1992; Arends et al., 1995; Giannoudis et al, 1999). The differences between these United Kingdom-based studies may reflect differences in HPV typing methods, and/or chance, and do not suggest significant variations between countries in the contribution of HPV 16 and/or 18 to cervical cancer incidence that are likely to have important effect on the impact of immunisation.

Our results are consistent with previous suggestions that a higher proportion of disease in Europe will be preventable by current HPV 16/18 vaccines than some other areas of the world (Muñoz et al, 2004; Li et al, 2010), mostly because of higher HPV 16 prevalence. Furthermore, the most common non-vaccine types identified in our study (HPV 33, 45, 52 and 31) were among the most common types found in international studies, albeit not in exactly the same ranking (Clifford et al, 2003; Muñoz et al, 2004; Li et al, 2010).

Several studies have reported data on HPV prevalence in women attending for cervical screening in the United Kingdom (Cuschieri et al, 2004; Peto et al, 2004; Kitchener et al, 2006; Hibbitts et al, 2008). In England, HR HPV prevalence of $16 \%$ (samples collected between 2001 and 2003) (Kitchener et al, 2006) and 7\% (samples collected between 1988 and 1993) (Peto et al, 2004) have previously been reported from studies conducted in Manchester. In south Wales (2008), HR HPV prevalence of $11 \%$ has been reported (Hibbitts et al, 2008), while in Scotland (2004), HR HPV prevalence of $16 \%$ has been reported (Cuschieri et al, 2004). In our study, the weighted (by age and cytology grade) prevalence of HR HPV was $16 \%(95 \%$ CI 11-22). These studies from across the United Kingdom also report fairly consistent results with respect to the prevalence of HPV 16 and/or 18. Differences in the age range, HPV typing methods, sample types and chance variation may account for some differences between studies. Differences may also reflect increases in prevalence over time, as the lowest HR HPV prevalence $(7 \%)$ was found in the earliest study (Peto et al, 2004 ), over a period when there has been an increasing frequency of clinically apparent HPV 6 and 11 infection as cases of genital warts (Health Protection Agency, 2008).

Strengths of our study include collection of both LBC samples and biopsy samples from a number of sites geographically spread across England, and testing for the same HPV types. The stratified sampling of LBCs from each centre enabled the description of type-specific infection in more severe cytology abnormalities and from older women.

This was a cross-sectional study of a disease state that spans a long time course of progression from infection to cancer: when comparing the type distribution in cancers and in lower grade disease we cannot therefore rule out any affect from the profile of HPV types in the population changing over time. Unfortunately, data on ethnicity, deprivation and sexual behaviour were not available with our samples and therefore the association between these factors and HPV prevalence could not be analysed.

Our estimates of prevalence are based on cervical screening. As repeat screens from individuals were not excluded and may be expected to have a higher HPV prevalence, our estimates may overestimate population prevalence among screened women (as do those from other similar studies of cervical screens). Our prevalence estimates do not provide data on the $20 \%$ of the population who do not regularly attend for screening (The NHS Information Centre, 2009), and who would have more to gain from immunisation.

This is the first study to describe comprehensively type-specific HPV prevalence across England, including samples from women with normal cytology and all stages of cervical disease in order to be able to estimate the proportion of disease potentially preventable, nationally, by immunisation. These data will be used in models to assess the likely benefit from HPV immunisation (Jit et al, 2008; Choi et al, 2009), and as baseline data against which to evaluate changes in type-specific HPV prevalence and type distribution after the introduction of the HPV immunisation programme. The findings support optimism regarding high impact of the National HPV (16/18) Immunisation Programme on cervical disease in England.

\section{ACKNOWLEDGEMENTS}

We gratefully acknowledge the 'NHSCSP HPV Special Interest Group' for their advice throughout the study. We also thank Mark Jit for helpful comments on the analyses and paper, and the reviewers of the paper. This study was funded by a grant from the NHS Cervical Screening Programme. RH-J and NdS were funded by the Research and Development Directorate of the United Kingdom Department of Health, grant reference number 039/030. The study was given a favourable ethical opinion by the South East MREC (reference 06/MRE01/48).

Supplementary Information accompanies the paper on British Journal of Cancer website (http://www.nature.com/bjc)

\section{REFERENCES}

Arends MJ, Donaldson YK, Duvall E, Wyllie AH, Bird CC (1993) Human papillomavirus type 18 associates with more advanced cerival neoplasia than human papillomavirus type 16. Hum Pathol 24: 432-437

Bouvard V, Baan R, Straif K, Grosse Y, Secretan B, El Ghissassi F, Benbrahim-Tallaa L, Guha N, Freeman C, Galichet L, Cogliano V, WHO International Agency for Research on Cancer Monograph Working Group (2009) A review of human carcinogens-Part B: biological agents. Lancet Oncol 10: $321-322$

Brown DR, Kjaer SK, Sigurdsson K, Iversen OE, Hernandez-Avila M, Wheeler CM, Perez G, Koutsky LA, Hseon Tay E, Garcia P, Ault KA, Garland SM, Leodolter S, Olsson SE, Tang GWK, Ferris DG, Paavonen J, Steben M, Bosch FX, Dillner J, Joura EA, Kurman RJ, Majewski S, Muñoz N, Myers ER, Villa LL, Taddeo FJ, Roberts C, Tadesse A, Bryan J,
Lupinacci LC, Giacoletti KED, Sings HL, James M, Hesley TM, Barr E (2009) The impact of quadrivalent human papillomavirus (HPV; types 6, 11,16 , and 18) L1 virus-like particle vaccine on infection and disease due to oncogenic nonvaccine HPV types in generally HPV-naive women aged 16-26 years. I Infect Dis 199: 926-935

Cancer Research UK (2009) Cervical cancer: UK cervical cancer statistics. May; Available from: http://info.cancerresearchuk.org/cancerstats/types/ cervix/?a $=5441$

Choi YH, Jit M, Gay N, Cox A, Garnett GP, Edmunds WJ (2009) Transmission dynamic modelling of the impact of human papillomavirus vaccination in the United Kingdom. Vaccine 28: 4091-4102

Chow S, Rodgers P (2005) Constructing area-proportional Venn and Euler diagrams with three circles. Euler Diagrams Workshop, Paris http:// www.cs.kent.ac.uk/people/staff/pjr/EulerVennCircles/EulerVennApplet.html 
Clifford GM, Smith JS, Plummer M, Franceschi S (2003) Human papillomavirus types in invasive cervical cancer worldwide: a meta-analysis. Br J Cancer 88: 63-73

Crook T, Wrede D, Tidy JA, Mason WP, Evans DJ, Vousden KH (1992) Clonal p53 mutation in primary cervical cancer: association with human-papillomavirus-negative tumours. Lancet 339: 1070-1073

Cuschieri KS, Brewster DH, Williams ARW, Millan D, Murray G, Nicoll S, Imrie J, Hardie A, Graham C, Cubie HA (2010) Distribution of HPV types associated with cervical cancers in Scotland and implications for the impact of HPV vaccines. Br J Cancer 102: 930-932

Cuschieri KS, Cubie HA, Whitley MW, Seagar AL, Arends MJ, Moore C, Gilkisson G, McGoogan E (2004) Multiple high risk HPV infections are common in cervical neoplasia and young women in a cervical screening population. J Clin Pathol 57: 68-72

Cuzick J, Terry G, Ho L, Monaghan J, Lopes A, Clarkson P, Duncan I (2000) Association between high-risk HPV types, HLA DRB1* and DQB1* alleles and cervical cancer in British women. Br J Cancer 82: 1348-1352

Giannoudis A, Graham DA, Southern SA, Herrington CS (1999) p53 codon $72 \mathrm{ARG}$ /PRO polymorphism is not related to HPV type or lesion grade in low- and high-grade squamous intra-epithelial lesions and invasive squamous carcinoma of the cervix. Int J Cancer 83: 66-69

Health Protection Agency (2008) Sexually Transmitted Infections and Young People in the United Kingdom: 2008 Report. Health Protection Agency: UK

Hibbitts S, Jones J, Powell N, Dallimore N, McRea J, Beer H, Tristram A, Fielder H, Fiander AN (2008) Human papillomavirus prevalence in women attending routine cervical screening in South Wales, UK: a crosssectional study. Br J Cancer 99: 1929-1933

Jit M, Choi YH, Edmunds WJ (2008) Economic evaluation of human papillomavirus vaccination in the United Kingdom. $\mathrm{Br}$ Med J 337: a769

Kitchener HC, Almonte M, Wheeler P, Desai M, Gilham C, Bailey A, Sargent A, Peto J on on behalf of the ARTISTIC Trial Study Group (2006) HPV testing in routine cervical screening: cross sectional data from the ARTISTIC trial. Br J Cancer 95: 52-61

Li N, Franceschi S, Howell-Jones R, Snijders PJF, Clifford GM (2010) Human papillomavirus type distribution in 30848 invasive cervical

\section{APPENDIX}

\section{Study Group Collaborators}

Andrew Bailey, Henry Kitchener (Chief Investigator), Alex Sargent (Central Manchester NHS Foundation Trust); Simon Beddows, Catherine Lowndes, John Parry, Kate Soldan, (HPA); Phil Bullock (Gloucestershire Hospitals NHS Foundation Trust); Paul Cross, cancers worldwide: variation by geographical region, histological type and year of publication. Int J Cancer (in press). doi:10.1002/ijc25396

Muñoz N, Bosch FX, Castellsague X, Diaz M, De Sanjose S, Hammouda D, Shah KV, Meijer CJLM (2004) Against which human papillomavirus types shall we vaccinate and screen? The international Perspective. Int J Cancer 111: 278-285

Office for National Statistics (2004) Office for National Statistics Census 2001: Key Statistics for Postcode Sectors in England and Wales. Crown Copyright. HMSO: London. ISBN 0116217499

Paavonen J, Naud P, Salmerón J, Wheeler CM, Chow S-N, Apter D, Kitchener H, Castellsague X, Teixeira JC, Skinner SR, Hedrick J, Jaisamrarn U, Limson G, Garland S, Szarewski A, Romanowski B, Aoki FY, Schwarz TF, Poppe WAJ, Bosch FX, Jenkins D, Hardt K, Zahaf T, Descamps D, Struyf F, Lehtinen M, Dubin G, for the HPV PATRICIA Study Group (2009) Efficacy of human papillomavirus (HPV)-16/18 AS04-adjuvanted vaccine against cervical infection and precancer caused by oncogenic HPV types (PATRICIA): final analysis of a double-blind, randomised study in young women. Lancet 374: $301-314$

Peto J, Gilham C, Deacon J, Taylor C, Evans C, Binns W, Haywood M, Elanko N, Coleman D, Yule R, Desai M (2004) Cervical HPV infection and neoplasia in a large population-based prospective study: the Manchester cohort. Br J Cancer 91: 942 -953

Powell N, Boyde A, Tristram A, Hibbitts S, Fiander A (2009) The potential impact of human papillomavirus vaccination in contemporary cytologically screened populations may be underestimated: an observational retrospective analysis of invasive cervical cancers. Int J Cancer 125: $2425-2427$

Sasieni P, Adams J, Cuzick J (2003) Benefit of cervical screening at different ages: evidence from the UK audit of screening histories. $\mathrm{Br} J$ Cancer 89: $88-93$

Tawfik El-Mansi M, Cuschieri KS, Morris RG, Williams AR (2006) Prevalence of human papillomavirus types 16 and 18 in cervical adenocarcinoma and its precursors in Scottish patients. Int J Gynecol Cancer 16: $1025-1031$

The NHS Information Centre (2009) Cervical Screening Programme England 2008-09. The Health and Social Care Information Centre: UK

Martin Jones, Trudy Johnson (Gateshead Health NHS Foundation Trust); Ray Lonsdale, Richard Wood, Carol Taylor (Norfolk and Norwich University Hospitals NHS Foundation Trust); Julietta Patnick (NHSCSP); Naveena Singh (Barts and the London NHS Trust); John Smith (Sheffield Teaching Hospitals NHS Foundation Trust); Christine Waddell, Raji Ganesan, Linda Bentley, Jane Adams (Birmingham Women's NHS Foundation Trust); Martin Young, John McGloin (Royal Free Hampstead NHS Trust). 